\title{
Generalizing and Integrating TOPSIS and Cook-Seiford Method for Multicriteria Group Decision-Making with Both Cardinal and Ordinal Data
}

\author{
Wu Li, ${ }^{1}$ Guanqi Guo, ${ }^{1}$ and Xiaoqiang $\mathrm{Zhou}^{2}$ \\ ${ }^{1}$ School of Information and Communication Engineering, Hunan Institute of Science and Technology, Yueyang 414006, China \\ ${ }^{2}$ School of Computer, Hunan Institute of Science and Technology, Yueyang 414006, China
}

Correspondence should be addressed to Wu Li; liwu0817@163.com

Received 5 December 2014; Accepted 21 February 2015

Academic Editor: Tofigh Allahviranloo

Copyright (C) $2015 \mathrm{Wu} \mathrm{Li}$ et al. This is an open access article distributed under the Creative Commons Attribution License, which permits unrestricted use, distribution, and reproduction in any medium, provided the original work is properly cited.

\begin{abstract}
The TOPSIS and Cook-Seiford social choice function are generalized and integrated for multicriteria group decision-making (MCGDM) with both cardinal evaluations and ordinal preferences of the alternatives. Unlike traditional TOPSIS, at first, the group's positive ideal solution and negative ideal solution under cardinal and ordinal preferences are defined, respectively. Thus the group rankings of the alternatives with respect to each criterion are derived from the individual preferences by the modified group TOPSIS considering the weights of decision makers under each criterion. Then the weighted distance function representing the total inconsistency between the comprehensive rankings of all alternatives and the ones under all criteria is presented after the criteria weights are taken into account. Form the perspective of minimizing the criteria-weighted distance of the rankings, a nonlinear integer programming is developed and transformed into an assignment problem to obtain the final rankings of all alternatives. An illustrative case is presented and some comparisons on the results show that the developed approach is practical and effective. This study extends TOPSIS to group decision-making with ordinal preferences and generalizes Cook-Seiford social choice function to multicriteria decision-making considering the criteria weights and can be a novel benchmark for MCGDM with both cardinal and ordinal data.
\end{abstract}

\section{Introduction}

Multicriteria group decision-making (MCGDM) has wide applications in engineering, economics, management, military fields, and so on. Its essence is assembling decision-making information, sorting, and selecting the outcome through definite alternatives by a group of decision makers according to multiple criteria [1-4]. As a typical MCGDM problem, for example, supplier selection in supply chain management needs to take into account multiple criteria including quality, delivery, performance history, warranties, price, technical capability, and financial position [5] and is usually made by multiple experts in quality control, financial management or supply chain management, and related administrative officials.

MCGDM often requires the experts to provide their qualitative/quantitative assessments for determining the performance of each alternative with respect to each criterion and thus leads to the concurrent appearance of both cardinal and ordinal preferences in the same MCGDM. For example, the experts usually are willing or easy to give their cardinal/ordinal preferences of alternatives for those qualitative/ quantitative criteria. Also, it is quite natural to think that different experts can provide their evaluations by means of such different preference structures as preference orderings, utility functions, multiplicative preference relations, fuzzy preference relations, and so on [6,7]. For group decision-making (GDM), fortunately, Chiclana and Delgado initiated a notable model with multiple preferences represented by means of preference orderings, utility functions, and fuzzy preference relations early in the late $1990 \mathrm{~s}[8,9]$. Following them, Herrera et al. [10], Mata et al. [11], Dong [6, 12], Fan et al. [13], Wang and Parkan [14], and Xu et al. [15] have made great progress in the GDM with different preference structures. Their methodologies can be divided into two categories: transformation function [8-12] and direct aggregation [6, 13-15] method. 
The former transforms all preferences in different structures into a uniform form and then aggregates the preferences unified to obtain the rankings of all alternatives. Unlike the transformation method, the latter obtain directly the rankings of all alternatives by developing and solving some optimization models [13-15] or aggregating the individual preferences determined firstly and separately. In spite of their success in dealing with the complexity resulting from multiple preference form, the transformation method may lose decision information and even cause preference distortion, and in the meanwhile, the direct framework is a bit convoluted [6]. Moreover, almost all these works have not involved multiple criteria; that is, little attention has been paid to MCGDM, especially with both cardinal and ordinal preferences. In fact, the technique for order performance by similarity to ideal solution (TOPSIS) [16] and Cook-Seiford social choice function [17] can be extended and combined for this problem, and this is just what we shall investigate in this paper.

As a useful technique in dealing with multicriteria decision-making (MCDM), TOPSIS argues that the ranking of alternatives will be based on the shortest distance from the positive ideal solution (PIS) and the farthest from the negative ideal solution (NIS). It simultaneously considers the distances to both PIS and NIS, and a preference order is ranked according to their relative closeness and a combination of these two distance measures [16]. According to Shih et al. [18], TOPSIS has advantages such as (i) a sound logic that represents the rationale of human choice; (ii) a scalar value that accounts for both the best and worst alternatives simultaneously; (iii) a simple computation process that can be easily programmed into a spreadsheet; and (iv) the performance measures of all alternatives on attributes which can be visualized on a polyhedron, at least for any two dimensions. Moreover, Zanakis et al. used the simulation comparison to show that TOPSIS has the fewest rank reversals among the eight methods in the category [19]. These advantages mentioned above make TOPSIS a major MCDM technique as compared with other related techniques such as AHP and ELECTRE. In recent years, TOPSIS has been extended to GDM problems with interval data, fuzzy data, or linguistic data [20-25]. These works generalizing TOPSIS for GDM can be classified into two categories: external aggregation and internal aggregation [18]. Outside the traditional TOPSIS procedure, the former utilizes some operations to manipulate the alternative ratings and weight ratings $[20,21]$ or uses a social welfare function to obtain a final ranking from individual decision makers of the group [22]. The latter aggregates the preference of individuals within the TOPSIS procedure [23]. Besides, in the external aggregation class, we can further distinguish the methods as preoperation (i.e., mathematical operators for cardinal information; refer to $[20,21]$ ) and postoperation (i.e., the Borda count or function for ordinal information; see [2]), which depend on whether the aggregation is done before or after the TOPSIS procedure. It seems that external aggregation aims to provide more information to support a complex decision, and the internal aggregation focuses on an integrated decision-making procedure.

In spite of the success of TOPSIS in GDM, there are several problems we need to discuss further. Firstly, these above works based on TOPSIS only dealt with the decision-making problem with crisp numbers, fuzzy data, interval data, or linguistic information of alternatives. How to extend TOPSIS to decision-making with ordinal preferences has remained unknown. Secondly, these works either used mathematical operators to manipulate the alternative ratings and weight ratings given by individual decision makers before the TOPSIS procedure or calculated the separation measures of PIS and NIS for the group after obtaining the separation measures from individual positive ideal solution (IPIS) and individual negative ideal solution (INIS) within the TOPSIS procedure. Like traditional TOPSIS, these works were based on IPIS and INIS rather than group positive ideal solution (GPIS) and group negative ideal solution (GNIS). Thirdly, these works only considered the whole weights of the decision makers without taking into account the weights of them under each criteria. For the same criteria, in fact, the authoritativeness and validity of evaluations of alternatives given by different decision maker may be different since the knowledge, ability, position, and familiarity with the decision-making problem of different decision maker are different. Thus, the weights of decision makers with respect to each criteria have to be considered, respectively. Lastly, it should be noted that traditional TOPSIS doubles the effects of attribute weighting on the separation measures. Thus, the priorities of alternatives are overly controlled by attribute weights. For these reasons, we shall modify and generalize TOPSIS for MCGDM with both cardinal and ordinal preferences.

As a good social choice method, Cook and Seiford [17] first defined a distance function on the set of all preference orders given by multiple decision makers and which was to be proved to satisfy certain desirable properties. Then they developed a nonlinear integer programming minimizing the distance between the final rankings of the alternatives and the ones given by all decision makers and transformed it to an assignment problem to obtain the rankings of the alternatives for the group. However, the method was used for GDM in single criteria setting. In fact, the CookSeiford method, although proposed initially for aggregating individual preferences in GDM, can be extended for MCDM to integrating the rankings of all alternatives under each criterion into the comprehensive rankings. More importantly, it regarded the sum of the difference of every alternative's ranking in two preference orders as the distance of the two preference orders and did not consider the decision makers' weights. So the method proposed by Cook and Seiford may yield multiple results for the same decision-making problem. In fact, its distance measurement approach can be modified.

For these reasons above, in this paper we propose a novel methodology for MCGDM with both cardinal and ordinal data. Firstly, the GPIS and GNIS with respect to each criterion under cardinal and ordinal preferences are defined, respectively, and then the alternatives' rankings for the group under each criterion are derived by our modified group TOPSIS. Secondly, considering all the criteria weights, we present a distance function to denote the inconsistency between the comprehensive rankings of all alternatives and the ones under all criteria. According to the idea that the ideal comprehensive ranking is the one which minimizes 
the criteria-weighted distance between itself and the ones under all criteria, a nonlinear integer programming is developed. We transform it into an assignment problem to obtain the final rankings of all alternatives. Lastly, an illustrative case is presented and some comparisons on the results show that the developed approach is practical and effective.

The remainder of this paper is organized as follows. The proposed approach is introduced in Section 2. Section 3 presents an illustrative case and some discussions on the results. Finally, several conclusions will be drawn in Section 4 .

\section{Proposed Framework}

In this section we shall develop a novel hybrid MCGDM model based on the combination of the extended TOPSIS and Cook-Seiford social choice function in the presence of both cardinal evaluations and ordinal preferences. Before presenting the proposed method we define and formulate the MCGDM first.

2.1. The MCGDM Problem Formulation. Without loss of generality and for the sake of simplicity, we put forward two reasonable assumptions as follows.

Firstly, we assume the individual cardinal preferences of the alternatives are expressed by crisp numbers since our proposed approach can also work well for other cardinal preference forms (e.g., interval data, fuzzy data, linguistic information, and so on) as long as the following group TOPSIS is modified accordingly.

Secondly, the criteria evaluated by cardinal preferences are set as benefit type and the cardinal evaluations have been standardized. Otherwise, they can be transformed into benefit indexes by some appropriate normalization method.

So we consider a MCGDM problem with $L$ decision makers evaluating $M$ alternatives according to $N$ criteria. $D_{l}(l=1,2, \ldots, L)$ is decision maker, $A_{m}(m=1,2, \ldots$, $M)$ is alternative, and $C_{n}(n=1,2, \ldots, N)$ is criteria. $\omega_{n}(n=1,2, \ldots, N)$ is the weight of criteria $C_{n}$ which satisfy $\sum_{n=1}^{N} \omega_{n}=1$ and $\omega_{n}>0 . \lambda_{n}^{l}(l=1,2, \ldots, L ; n=1,2, \ldots, N)$ is the weight of decision maker $D_{l}$ under criteria $C_{n}$ which satisfy $\sum_{l=1}^{L} \lambda_{n}^{l}=1$ and $\lambda_{n}^{l}>0 . r_{n m}^{l}$ is the performance rating of alternative $A_{m}$ with respect to criteria $C_{n}$ given by decision maker $D_{l}$. For cardinal evaluations $r_{n m}^{l}$ is a crisp number and for ordinal preferences it is the rank position of $A_{m}$ and $r_{n m}^{l} \in\{1,2, \ldots, M\}$. For certain $n(n=1,2, \ldots, N)$, all $r_{n m}^{l}$ constitute the group decision matrix $R_{n}=\left[r_{n m}^{l}\right]_{M \times L}$ as follows:

$$
R_{n}=\left[\begin{array}{cccc}
r_{n 1}^{1} & r_{n 1}^{2} & \cdots & r_{n 1}^{L} \\
r_{n 2}^{1} & r_{n 2}^{2} & \cdots & r_{n 2}^{L} \\
\vdots & \vdots & & \vdots \\
r_{n M}^{1} & r_{n M}^{2} & \cdots & r_{n M}^{L}
\end{array}\right]
$$

Below our concern is to obtain the final rankings of all alternatives in the opinion of the group of the decision makers.
2.2. The Proposed Algorithm. To obtain the final rankings of all alternatives for the group, there are two processes to implement, namely, (1) the aggregation process for aggregating the individual preferences under each criterion into group rankings of the alternatives by modified group TOPSIS and (2) the integration process for integrating the group rankings of the alternatives under each criterion into the final comprehensive rankings of them by generalized CookSeiford method.

(1) The Aggregation Process Based on Modified Group TOPSIS. We present firstly the concepts of GPIS and GNIS as follows.

Definition 1. If the performance ratings of an alternative with respect to certain criteria $C_{n}$ given by all decision makers are all the highest one, then the alternative is the GPIS with respect to criteria $C_{n}$, named GPIS $_{n}$.

Definition 2. If the performance ratings of an alternative with respect to certain criteria $C_{n}$ given by all decision makers are all the lowest one, then the alternative is the GNIS with respect to criteria $C_{n}$, named GNIS $_{n}$.

Now we detail the proposed modified group TOPSIS. The algorithm involves the following steps.

Step 1. Determine $\mathrm{GPIS}_{n}$ and $\mathrm{GNIS}_{n}$ under criteria $C_{n}(n=$ $1,2, \ldots, N)$ :

$$
\begin{aligned}
\mathrm{GPIS}_{n} & =\left(r_{n}^{1+}, r_{n}^{2+}, \ldots, r_{n}^{L+}\right), \\
\mathrm{GNIS}_{n} & =\left(r_{n}^{1-}, r_{n}^{2-}, \ldots, r_{n}^{L-}\right),
\end{aligned}
$$

where

$$
\begin{aligned}
& r_{n}^{l+}= \begin{cases}\max _{m} r_{n m}^{l}, & \text { for cardinal evaluations; } \\
1, & \text { for ordinal preferences }\end{cases} \\
& l=1,2, \ldots, L . \\
& r_{n}^{l-}= \begin{cases}\min _{m} r_{n m}^{l}, & \text { for cardinal evaluations; } \\
M, & \text { for ordinal preferences }\end{cases} \\
& l=1,2, \cdots, L .
\end{aligned}
$$

Step 2. Calculate $d_{n m}^{+}$and $d_{n m}^{-}$, respectively, that is, the separation measure of $A_{m}(m=1,2, \ldots, M)$ from GPIS $_{n}$ and GNIS $_{n}$ under criteria $C_{n}(n=1,2, \ldots, N)$ for the group

$$
\begin{aligned}
& d_{n m}^{+}=\sqrt{\sum_{l=1}^{L} \lambda_{n}^{l}\left(r_{n m}^{l}-r_{n}^{l+}\right)^{2}}, \\
& d_{n m}^{-}=\sqrt{\sum_{l=1}^{L} \lambda_{n}^{l}\left(r_{n m}^{l}-r_{n}^{l-}\right)^{2}} .
\end{aligned}
$$

Note. The traditional TOPSIS doubles the effects of attribute weighting on the separation measures. Thus, the priorities of 
alternatives are overly controlled by attribute weights. So we adopt the weighted Minkowski distance function above to overcome this problem and also to enhance the reliability of decision.

Step 3. Calculate $d_{n m}$, the relative closeness of $A_{m}(m=1,2$, $\ldots, M)$ to $\mathrm{GPIS}_{n}(n=1,2, \ldots, N)$ under criteria $C_{n}$ for the group

$$
d_{n m}=\frac{d_{n m}^{-}}{d_{n m}^{-}+d_{n m}^{+}} .
$$

Step 4. Obtain $r_{n m}^{G}$, the group ranking position of $A_{m}(m=$ $1,2, \ldots, M)$ under criteria $C_{n}(n=1,2, \ldots, N)$. We can rank the alternatives using $d_{n m}$ in descending order. In other words, the larger $d_{n m}$ is, the more superior $A_{m}$ is with respect to criteria $C_{n}$ for the group.

(2) The Integration Process Based on Generalized Cook-Seiford Method. Cook-Seiford social choice function calculates firstly the sum of the differences of each alternative' ranking position between the group and a certain individual ordering vector without considering decision maker weight and regards the sum as the inconsistency of the group preference and the individual rankings. Then it adds up all these sums to obtain the total inconsistency of the group and all individual ordinal preferences. Inspired by the Cook-Seiford method and unlike it, we first consider the criteria weights and calculate the weighted deviation between the comprehensive ranking position and the one under all criteria of single alternative (not ordering vector) then add up the weighted deviations of all alternatives to the total inconsistency between the comprehensive rankings of all alternatives and the ones under all criteria as follows.

Definition 3. The weighted distance representing the total inconsistency between the comprehensive rankings of all alternatives and the ones under all criteria is expressed by $\sum_{m=1}^{M} \sqrt{\sum_{n=1}^{N} \omega_{n}\left(r_{m}^{G}-r_{n m}^{G}\right)^{2}}$, where $r_{m}^{G}$ is the final group ranking position of $A_{m}(m=1,2, \ldots, M)$.

Now the integration process is composed of the following two steps.

Step 1. Develop the optimization model to determine $r_{m}^{G}$, the final ranking position of $A_{m}(m=1,2, \ldots, M)$.

As mentioned above, the comprehensive rankings of all alternatives should minimize the weighted distance in Definition 3. So the final rankings of them in the view of the group can be obtained by solving a nonlinear integer programming as follows:

$$
\begin{array}{ll}
\left(P_{1}\right) \min & \sum_{m=1}^{M} \sqrt{\sum_{n=1}^{N} \omega_{n}\left(r_{m}^{G}-r_{n m}^{G}\right)^{2}} \\
\text { s.t. } & r_{m}^{G} \in\{1,2, \ldots, M\} \quad(m=1,2, \ldots, M) \\
& r_{j}^{G} \neq r_{k}^{G} \quad(j \neq k ; j, k=1,2, \ldots, M) .
\end{array}
$$

TABLE 1: The weights of all criteria and decision makers under each criterion.

\begin{tabular}{ccccccc}
\hline$n$ & $\omega_{n}$ & $\lambda_{n}^{1}$ & $\lambda_{n}^{2}$ & $\lambda_{n}^{3}$ & $\lambda_{n}^{4}$ & $\lambda_{n}^{5}$ \\
\hline 1 & 0.3 & 0.3 & 0.15 & 0.15 & 0.15 & 0.25 \\
2 & 0.3 & 0.15 & 0.3 & 0.25 & 0.15 & 0.15 \\
3 & 0.2 & 0.2 & 0.2 & 0.2 & 0.2 & 0.2 \\
4 & 0.2 & 0.2 & 0.2 & 0.2 & 0.2 & 0.2 \\
\hline
\end{tabular}

Step 2. Solve the optimization model above and obtain the $r_{m}^{G}$, the final ranking position of $A_{m}(m=1,2, \ldots, M)$.

Since the nonlinear integer programming above is hard to solve by traditional approach to nonlinear programming or integer programming, we have to turn to other methods. In fact, each alternative must be ranked at a location among 1 to $M$, and each alternative can be located only one location in the above range, so the final rankings of all alternatives can be obtained by solving the following assignment problem:

$$
\begin{aligned}
\left(P_{2}\right) \min & \sum_{j=1}^{M} \sum_{k=1}^{M} x_{j k} \sqrt{\sum_{n=1}^{N} \omega_{n}\left(k-r_{n j}^{G}\right)^{2}} \\
\text { s.t. } \quad & \sum_{k=1}^{M} x_{j k}=1 \quad(j=1,2, \ldots, M) \\
& \sum_{j=1}^{M} x_{j k}=1 \quad(k=1,2, \ldots, M) \\
& x_{j k} \in\{0,1\} \quad(j, k=1,2, \ldots, M) .
\end{aligned}
$$

$\sqrt{\sum_{n=1}^{N} \omega_{n}\left(k-r_{n j}^{G}\right)^{2}}$ in formula (7) denotes the weighted deviation between the comprehensive ranking position and the ones under all criteria of $S_{j}$ when its final ranking position is set at $k$. Formulas (8) and (10) indicate that each alternative can be located in only one location, and similarly formulas (9) and (10) say that each location can also be located by only one alternative. $x_{j k}=1(j, k=1,2, \ldots, M)$ in the solution of $\left(P_{2}\right)$ implies that $r_{j}^{G}=k$, that is to say, the final group ranking position of $S_{j}$, is $k$.

\section{Illustrative Case and Discussions on the Results}

In this section we utilize a supplier selection example in hydroelectric project supply chain to illustrate the application of the developed approach and then present some comparisons and analyses on the results.

3.1. An Illustrative Case. Consider a MCGDM problem with 5 decision makers evaluating 4 alternatives according to 4 criteria. The weights of the criteria and the decision makers with respect to each criterion are listed in Table 1.

Suppose the decision makers gave their cardinal evaluation for the first and second criteria, and ordinal preferences for the latter two criteria. The performance rating of alternatives with respect to each criteria given by the decision 
makers constitute the group decision making matrix $R_{n}(n=$ $1,2,3,4)$ as follows:

$$
\begin{aligned}
& R_{1}=\left[\begin{array}{lllll}
0.3 & 0.5 & 0.4 & 0.3 & 0.2 \\
0.6 & 0.3 & 0.3 & 0.7 & 0.5 \\
0.2 & 0.6 & 0.7 & 0.4 & 0.4 \\
0.7 & 0.5 & 0.6 & 0.5 & 0.6
\end{array}\right], \\
& R_{2}=\left[\begin{array}{lllll}
0.2 & 0.3 & 0.6 & 0.6 & 0.4 \\
0.4 & 0.7 & 0.9 & 0.4 & 0.8 \\
0.8 & 0.2 & 0.6 & 0.5 & 0.6 \\
0.6 & 0.8 & 0.3 & 0.8 & 0.5
\end{array}\right], \\
& R_{3}=\left[\begin{array}{lllll}
4 & 3 & 3 & 4 & 4 \\
3 & 2 & 1 & 2 & 2 \\
2 & 4 & 4 & 3 & 3 \\
1 & 1 & 2 & 1 & 1
\end{array}\right], \\
& R_{4}=\left[\begin{array}{lllll}
3 & 3 & 2 & 3 & 4 \\
2 & 1 & 4 & 1 & 1 \\
4 & 4 & 3 & 4 & 3 \\
1 & 2 & 1 & 2 & 2
\end{array}\right] .
\end{aligned}
$$

Now we use the developed approach to rank the alternatives and the procedure is as follows.

Step 1. Determine GPIS $n$ and GNIS $n$ where $n=1,2,3,4$,

$$
\begin{gathered}
\mathrm{GPIS}_{1}=(0.7,0.6,0.7,0.7,0.6), \\
\mathrm{GNIS}_{1}=(0.2,0.3,0.3,0.3,0.2) ; \\
\mathrm{GPIS}_{2}=(0.8,0.8,0.9,0.8,0.8), \\
\mathrm{GNIS}_{2}=(0.2,0.2,0.3,0.4,0.4) ; \\
\text { GPIS }_{3}=(1,1,1,1,1), \\
\text { GNIS }_{3}=(4,4,4,4,4) ; \\
\text { GPIS }_{4}=(1,1,1,1,1), \\
\text { GNIS }_{4}=(4,4,4,4,4) .
\end{gathered}
$$

Step 2. Calculate $d_{n m}$, the relative closeness of $A_{m}(m=$ $1,2,3,4)$ to $\mathrm{GPIS}_{n}(n=1,2,3,4)$. The results are listed in Table 2.

Step 3. Obtain $r_{n m}^{G}$, the group ranking position of $A_{m}(m=$ $1,2,3,4)$, under criteria $C_{n}(n=1,2,3,4)$. The result can be derived from the data in Table 2 and listed in Table 3.

Step 4. Determine $r_{m}^{G}$, the final group ranking position of $A_{m}(m=1,2,3,4)$.
TABLE 2: The relative closeness of all alternatives to the corresponding GPIS.

\begin{tabular}{lcccc}
\hline$m$ & $d_{1 m}$ & $d_{2 m}$ & $d_{3 m}$ & $d_{4 m}$ \\
\hline 1 & 0.223330 & 0.294086 & 0.192927 & 0.360645 \\
2 & 0.597171 & 0.661637 & 0.639355 & 0.637771 \\
3 & 0.413591 & 0.428023 & 0.320377 & 0.192927 \\
4 & 0.798026 & 0.545316 & 0.863473 & 0.759747 \\
\hline
\end{tabular}

TABLE 3: The rankings of all suppliers with respect to each criterion for the group.

\begin{tabular}{lcccc}
\hline$m$ & $r_{1 m}^{G}$ & $r_{2 m}^{G}$ & $r_{3 m}^{G}$ & $r_{4 m}^{G}$ \\
\hline 1 & 4 & 4 & 4 & 3 \\
2 & 2 & 1 & 2 & 2 \\
3 & 3 & 3 & 3 & 4 \\
4 & 1 & 2 & 1 & 1 \\
\hline
\end{tabular}

Let $e_{j k}=\sqrt{\sum_{n=1}^{N} \omega_{n}\left(k-r_{n j}^{G}\right)^{2}}(j, k=1,2,3,4)$ and then constitute $E=\left[e_{j k}\right]_{4 \times 4}$ where $e_{j k}$ is located in the $j$ th row and $k$ th column as follows:

$$
E=\left[\begin{array}{llll}
2.82843 & 1.84391 & 0.89443 & 0.44721 \\
0.83666 & 0.54772 & 1.37840 & 2.34521 \\
2.23607 & 1.26491 & 0.44721 & 0.89443 \\
0.54772 & 0.83666 & 1.51658 & 2.73861
\end{array}\right]
$$

Substituting $E$ into $\left(P_{2}\right)$, we obtain the solution:

$$
\begin{gathered}
x_{14}=x_{22}=x_{33}=x_{41}=1 \\
x_{j k}=0, \quad \text { for other } j, k .
\end{gathered}
$$

That is to say, the final group rankings of 4 alternatives are

$$
A_{4}>A_{2}>A_{3}>A_{1} \text {. }
$$

So the best alternative is $A_{4}$.

3.2. Discussions on the Results. To show the effectiveness of the proposed method, we shall solve the illustrative MCGDM problem again by other existing methods.

For the cardinal evaluations, at first, we can use weighted sum method to rank the alternatives for the group. According to the weighted sum method, we can set $e_{n m}^{G}=$ $\sum_{l=1}^{L} \lambda_{n m}^{l} r_{n m}^{l}(n=1,2 ; m=1,2,3,4)$ and then rank the alternatives using the index in descending order. In other words, the larger $e_{n m}^{G}$ is, the more superior $A_{m}$ is for the group. All $e_{n m}^{G}$ under the first and second criteria are as follows:

$$
\begin{array}{cccc}
e_{11}^{G}=0.32, & e_{12}^{G}=0.5, & e_{13}^{G}=0.415, & e_{14}^{G}=0.6 ; \\
e_{21}^{G}=0.42, & e_{22}^{G}=0.675, & e_{23}^{G}=0.495, & e_{24}^{G}=0.6 .
\end{array}
$$


So the rankings of the alternatives under these two criteria for the group are as follows:

$$
\begin{array}{llll}
r_{11}^{G}=4, & r_{12}^{G}=2, & r_{13}^{G}=3, & r_{14}^{G}=1 ; \\
r_{21}^{G}=4, & r_{22}^{G}=1, & r_{23}^{G}=3, & r_{24}^{G}=2 .
\end{array}
$$

Obviously the results are identical with the ones obtained by the proposed method above.

Secondly, we utilize the Borda count method [2] to obtain the rankings of the alternatives under the criteria evaluated by ordinal preferences. The Borda count is defined as $b_{n m}^{G}=$ $\sum_{l=1}^{L}\left(M-r_{n m}^{l}\right)(n=3,4 ; m=1,2,3,4)$ and similarly the larger $b_{n m}^{G}$ is, the more superior $A_{m}$ is for the group. Thus we have

$$
\begin{array}{llll}
b_{31}^{G}=2, & b_{32}^{G}=10, & b_{33}^{G}=4, & b_{34}^{G}=14 ; \\
b_{41}^{G}=5, & b_{42}^{G}=11, & b_{43}^{G}=2, & b_{44}^{G}=12 .
\end{array}
$$

So the rankings of the suppliers with respect to the criteria of supply capacity and after service for the group are as follows and they are also identical with the ones obtained by the proposed method above:

$$
\begin{array}{llll}
r_{31}^{G}=4, & r_{32}^{G}=2, & r_{33}^{G}=3, & r_{34}^{G}=1 ; \\
r_{41}^{G}=3, & r_{42}^{G}=2, & r_{43}^{G}=4, & r_{44}^{G}=1 .
\end{array}
$$

It must be noted that the Borda count above does not take into account the weights of the decision makers since the weights of them in the illustrative case are just the same. Otherwise, the weighted Borda count can be expressed as $b_{n m}^{G}=\sum_{l=1}^{L} \lambda_{n}^{l} \times\left(M-r_{n m}^{l}\right)(n=3,4 ; m=1,2,3,4)$ which shall bring about the same results.

Now we calculate the final rankings of all alternatives for the group by Bernardo method [2] which involves the following steps.

Step 1. Define the consistency matrix $F_{n}=\left[f_{j k}^{n}\right]_{M \times M}$ under criteria $C_{n}(n=1,2, \ldots, N)$ where

$$
f_{j k}^{n}= \begin{cases}1, & \text { when } r_{n j}^{G}=k ;(j, k=1,2, \ldots, M) \\ 0, & \text { otherwise. }\end{cases}
$$

Step 2. Calculate the weighted consistency matrix $F=$ $\left[f_{j k}\right]_{M \times M}=\sum_{n=1}^{N} \omega_{n} \times F_{n}$.

Step 3. Obtain $r_{m}^{G}$, the comprehensive ranking position of $A_{m}(m=1,2, \ldots, M)$ for the group by solving the following $0-1$ programming problem:

$$
\begin{aligned}
\left(P_{3}\right) \max & \sum_{j=1}^{M} \sum_{k=1}^{M} f_{j k} x_{j k} \\
\text { s.t. } & \sum_{k=1}^{M} x_{j k}=1, \quad j=1,2, \ldots, M
\end{aligned}
$$

$$
\begin{aligned}
& \sum_{j=1}^{M} x_{j k}=1, \quad k=1,2, \ldots, M \\
& x_{j k} \in\{0,1\}, \quad j, k=1,2, \ldots, M .
\end{aligned}
$$

$x_{j k}=1(j, k=1,2, \ldots, M)$ in the solution of $\left(P_{3}\right)$ indicates $r_{j}^{G}=k$; that is to say, the final ranking position of $A_{j}$ is $k$.

According to the procedure above, we have

$$
F=\left[\begin{array}{cccc}
0 & 0 & 0.2 & 0.8 \\
0.3 & 0.7 & 0 & 0 \\
0 & 0 & 0.8 & 0.2 \\
0.7 & 0.3 & 0 & 0
\end{array}\right]
$$

Substituting $F$ into $\left(P_{3}\right)$, we obtain the solution:

$$
\begin{gathered}
x_{14}=x_{22}=x_{33}=x_{41}=1 ; \\
x_{j k}=0, \quad \text { for other } j, k .
\end{gathered}
$$

The solution indicates that the final rankings of 4 alternatives in the view of the group are

$$
A_{4}>A_{2}>A_{3}>A_{1} .
$$

The final result is also identical with the one obtained by the proposed method in this paper and this just indicates that it is effective and rational.

\section{Conclusions}

In this paper we investigate the MCGDM problem with both cardinal and ordinal preferences and develop an approach based on combination of our extended group TOPSIS and generalized Cook-Seiford social choice method. The main contribution of this study may be summarized as follows.

(1) Group ideal solution concepts for cardinal and ordinal preferences are presented and thus a modified group TOPSIS without doubling the effects of attribute weighting on the separation measures is developed for MCGDM.

(2) A weighted distance function of ranking vectors is defined, and thus the Cook-Seirford social choice function is extended for MCGDM.

(3) The proposed framework can be a benchmark solution for MCGDM with both cardinal and ordinal preferences since it can work well for other cardinal preference forms as long as the group TOPSIS is modified accordingly.

However, we do not involve the situation where there are multiple different preference structures even under one criteria. These problems will be left for our future study.

\section{Conflict of Interests}

The authors declare that there is no conflict of interests regarding the publication of this paper. 


\section{Acknowledgments}

This work was supported by the National Natural Science Foundation of China (no. 61473118), the Natural Science Foundation of Hunan Province (no. 2015JJ2074), China Postdoctoral Science Foundation (no. 2012M511773), Social Science Foundation of Hunan Province (no. 2010YBA104), and Scientific Research Fund of Hunan Provincial Education Department (no. 13K102).

\section{References}

[1] Z.-C. Jiang and Y. Wang, "Multiattribute group decision making with unknown decision expert weights information in the framework of interval intuitionistic trapezoidal fuzzy numbers," Mathematical Problems in Engineering, vol. 2014, Article ID 635476, 7 pages, 2014.

[2] C. L. Hwang and M. J. Lin, Group Decision Making under Multiple Criteria: Methods and Applications, Springer, Berlin, Germany, 1987.

[3] S.-P. Wan and D.-F. Li, "Atanassov's intuitionistic fuzzy programming method for heterogeneous multiattribute group decision making with Atanassov's intuitionistic fuzzy truth degrees," IEEE Transactions on Fuzzy Systems, vol. 22, no. 2, pp. 300-312, 2014.

[4] P.-D. Liu and X.-C. Yu, "2-Dimension uncertain linguistic power generalized weighted aggregation operator and its application in multiple attribute group decision making," KnowledgeBased Systems, vol. 57, pp. 69-80, 2014.

[5] J. Chai, J. N. K. Liu, and E. W. T. Ngai, "Application of decisionmaking techniques in supplier selection: a systematic review of literature," Expert Systems with Applications, vol. 40, no. 10, pp. 3872-3885, 2013.

[6] Y.-C. Dong and H.-J. Zhang, "Multiperson decision making with different preference representation structures: a direct consensus framework and its properties," Knowledge-Based Systems, vol. 58, pp. 45-57, 2014.

[7] G.-W. Wei, "Grey relational analysis model for dynamic hybrid multiple attribute decision making," Knowledge-Based Systems, vol. 24, no. 5, pp. 672-679, 2011.

[8] M. Delgado, F. Herrera, E. Herrera-Viedma, and L. Martínezb, "Combining numerical and linguistic information in group decision making," Information Sciences, vol. 107, no. 1-4, pp. 177194, 1998.

[9] F. Chiclana, F. Herrera, and E. Herrera-Viedma, "Integrating three representation models in fuzzy multipurpose decision making based on fuzzy preference relations," Fuzzy Sets and Systems, vol. 97, no. 1, pp. 33-48, 1998.

[10] F. Herrera, L. Martínez, and P. J. Sánchez, "Managing nonhomogeneous information in group decision making," European Journal of Operational Research, vol. 166, no. 1, pp. 115-132, 2005.

[11] F. Mata, L. Martínez, and E. Herrera-Viedma, "An adaptive consensus support model for group decision-making problems in a multigranular fuzzy linguistic context," IEEE Transactions on Fuzzy Systems, vol. 17, no. 2, pp. 279-290, 2009.

[12] Y.-C. Dong, Y.-F. Xu, and S. Yu, "Linguistic multiperson decision making based on the use of multiple preference relations," Fuzzy Sets and Systems, vol. 160, no. 5, pp. 603-623, 2009.

[13] Z.-P. Fan, J. Ma, Y.-P. Jiang, Y.-H. Sun, and L. Ma, "A goal programming approach to group decision making based on multiplicative preference relations and fuzzy preference relations," European Journal of Operational Research, vol. 174, no. 1, pp. 311-321, 2006.

[14] Y.-M. Wang and C. Parkan, "Optimal aggregation of fuzzy preference relations with an application to broadband internet service selection," European Journal of Operational Research, vol. 187, no. 3, pp. 1476-1486, 2008.

[15] Z.-S. Xu, X.-Q. Cai, and S.-S. Liu, "Nonlinear programming model integrating different preference structures," IEEE Transactions on Systems, Man, and Cybernetics Part A:Systems and Humans, vol. 41, no. 1, pp. 169-177, 2011.

[16] C. L. Hwang and K. Yoon, Multiple Attribute Decision Making, Springer, Berlin, Germany, 1981.

[17] W. D. Cook and L. M. Seiford, "Priority ranking and consensus formation," Management Science, vol. 24, no. 16, pp. 1721-1732, 1978.

[18] H. S. Shih, H. J. Shyur, and E. S. Lee, "An extension of TOPSIS for group decision making," Mathematical and Computer Modelling, vol. 45, no. 7-8, pp. 801-813, 2007.

[19] S. H. Zanakis, A. Solomon, N. Wishart, and S. Dublish, "Multiattribute decision making: a simulation comparison of select methods," European Journal of Operational Research, vol. 107, no. 3, pp. 507-529, 1998.

[20] C.-T. Chen, "Extensions of the TOPSIS for group decisionmaking under fuzzy environment," Fuzzy Sets and Systems, vol. 114, no. 1, pp. 1-9, 2000.

[21] Y.-J. Wang and H.-S. Lee, "Generalizing TOPSIS for fuzzy multiple-criteria group decision-making," Computers \& Mathematics with Applications, vol. 53, no. 11, pp. 1762-1772, 2007.

[22] T.-C. Chu, "Facility location selection using fuzzy TOPSIS under group decisions," International Journal of Uncertainty, Fuzziness and Knowledge-Based Systems, vol. 10, no. 6, pp. 687701, 2002.

[23] Y.-H. Lin, P.-C. Lee, T.-P. Chang, and H.-I. Ting, "Multiattribute group decision making model under the condition of uncertain information," Automation in Construction, vol. 17, no. 6, pp. 792-797, 2008.

[24] F. Liu and W.-G. Zhang, "TOPSIS-based consensus model for group decision-making with incomplete interval fuzzy preference Relations," IEEE Transactions on Cybernetics, vol. 44, no. 8, pp. 1283-1294, 2013.

[25] Z.-L. Yue, “TOPSIS-based group decision-making methodology in intuitionistic fuzzy setting," Information Sciences, vol. 277, pp. 141-153, 2014. 


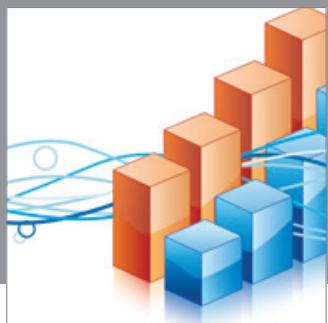

Advances in

Operations Research

mansans

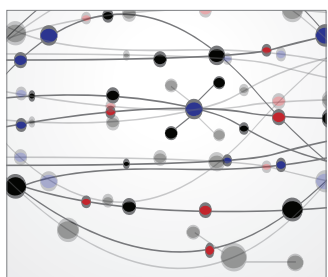

The Scientific World Journal
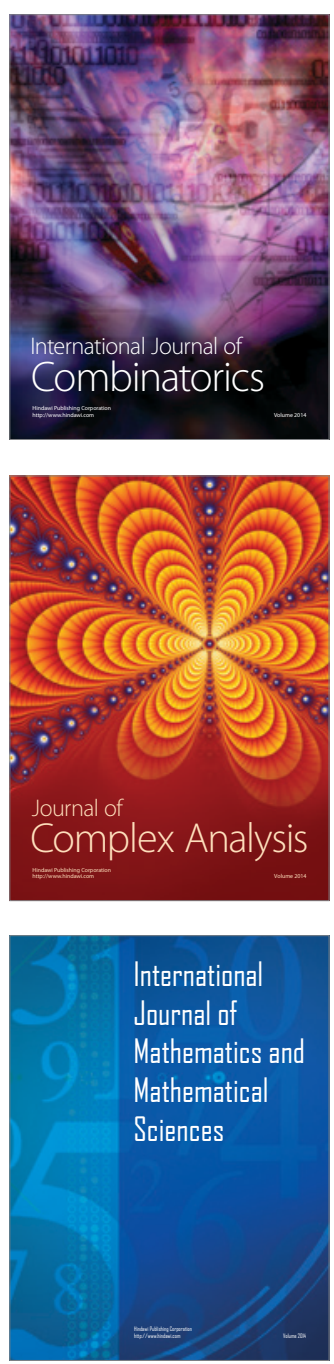
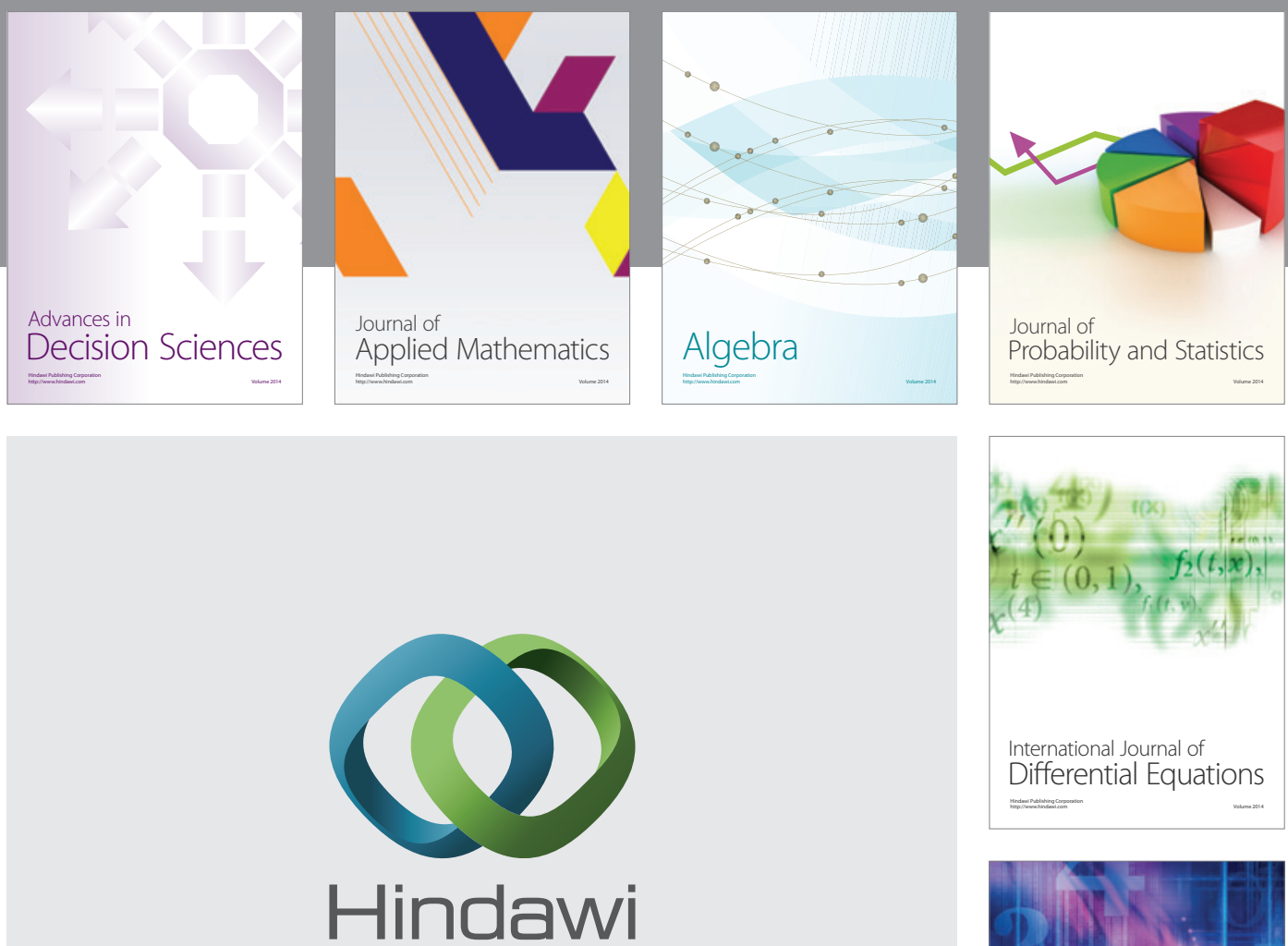

Submit your manuscripts at http://www.hindawi.com
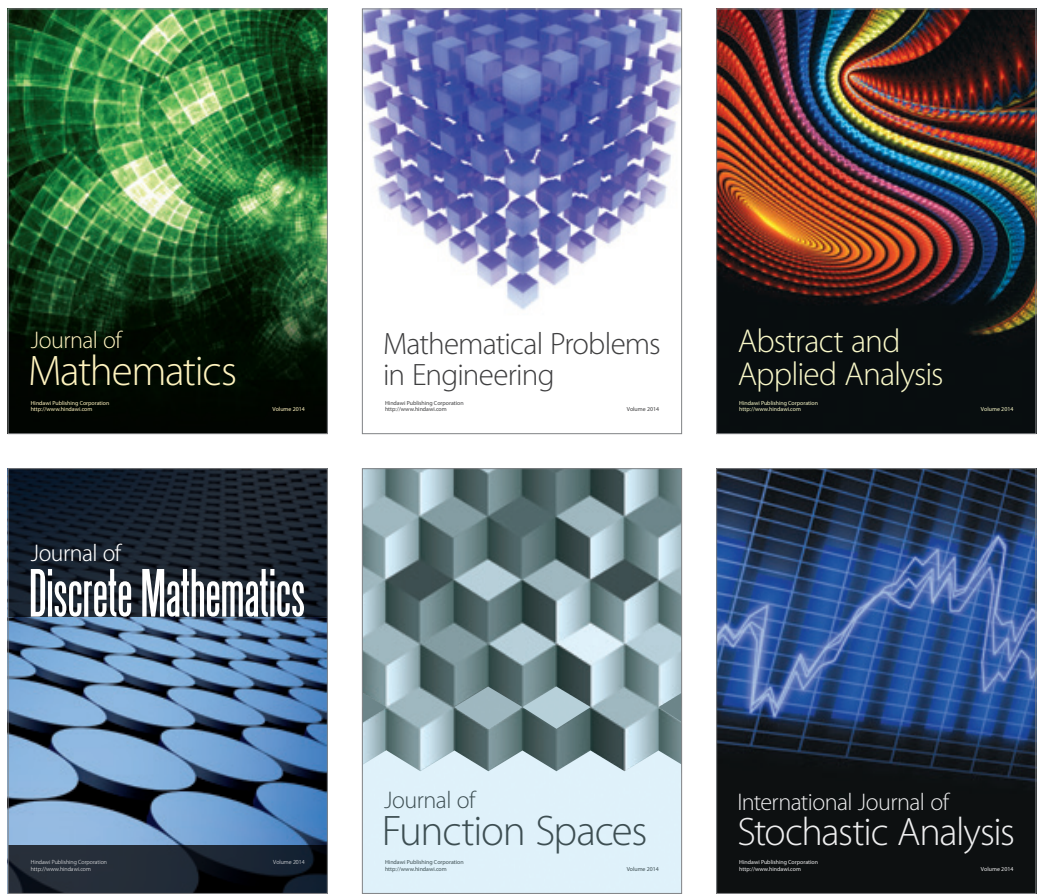

Journal of

Function Spaces

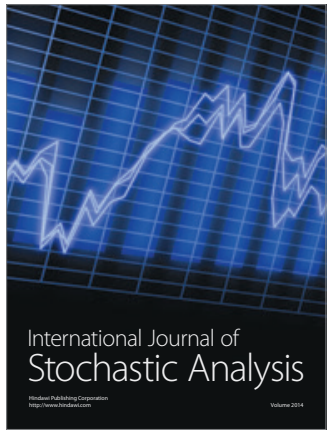

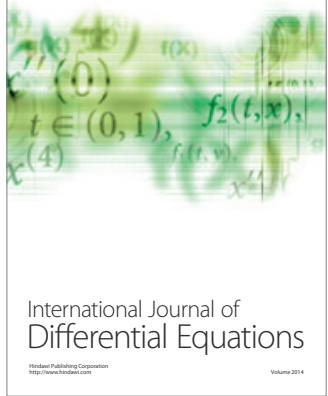
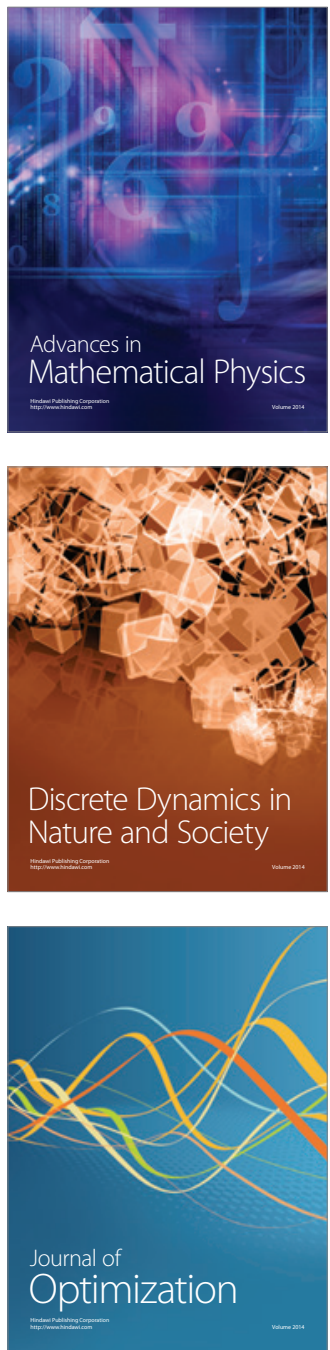